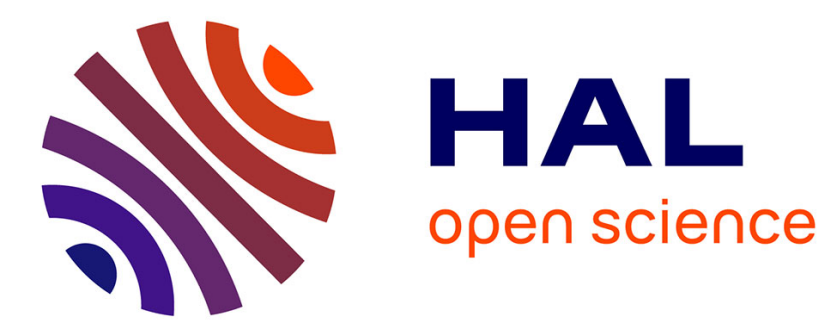

\title{
Nucléation par le borax du sulfate de sodium dispersé. application possible au stockage thermique
}

\author{
L. Babin, D. Clausse, I. Sifrini, F. Broto, M. Clausse
}

\section{To cite this version:}

L. Babin, D. Clausse, I. Sifrini, F. Broto, M. Clausse. Nucléation par le borax du sulfate de sodium dispersé. application possible au stockage thermique. Journal de Physique Lettres, 1978, 39 (20), pp.359-361. 10.1051/jphyslet:019780039020035900 . jpa-00231516

\section{HAL Id: jpa-00231516 https://hal.science/jpa-00231516}

Submitted on 1 Jan 1978

HAL is a multi-disciplinary open access archive for the deposit and dissemination of scientific research documents, whether they are published or not. The documents may come from teaching and research institutions in France or abroad, or from public or private research centers.
L'archive ouverte pluridisciplinaire HAL, est destinée au dépôt et à la diffusion de documents scientifiques de niveau recherche, publiés ou non, émanant des établissements d'enseignement et de recherche français ou étrangers, des laboratoires publics ou privés. 


\title{
NUCLÉATION PAR LE BORAX DU SULFATE DE SODIUM DISPERSÉ. APPLICATION POSSIBLE AU STOCKAGE THERMIQUE
}

\author{
L. BABIN, D. CLAUSSE, I. SIFRINI, F. BROTO et M. CLAUSSE
}

Laboratoire de Thermodynamique, Institut Universitaire de Recherche Scientifique Université de Pau et des Pays de l'Adour, avenue Philippon, 64000 Pau, France

(Reçu le 6 mars 1978, révisé le 12 juin 1978, accepté le 16 juin 1978)

\begin{abstract}
Résumé. - On a étudié par analyse enthalpique différentielle la cristallisation de gouttes de solutions aqueuses de $\mathrm{Na}_{2} \mathrm{SO}_{4}\left(0,9\right.$ mole $\mathrm{Na}_{2} \mathrm{SO}_{4} ; 10$ moles $\left.\mathrm{H}_{2} \mathrm{O}\right)$ contenant un excès de $\mathrm{Na}_{2} \mathrm{~B}_{4} \mathrm{O}_{7}$, $10 \mathrm{H}_{2} \mathrm{O}$ solide et dispersées dans un support émulsionnant. On observe deux maxima de cristallisations à $+7{ }^{\circ} \mathrm{C}$ et $-2,5^{\circ} \mathrm{C}$ au lieu de $-41^{\circ} \mathrm{C}$ en l'absence de borax. Entre le premier et trentième cycle de refroidissement-réchauffement on observe une augmentation de $15 \%$ de l'énergie libérée lors de la cristallisation. Ce type de nucléation pourrait présenter un intérêt pour la réalisation de stockage thermique.
\end{abstract}

\begin{abstract}
The crystallization of droplets of aqueous solutions of $\mathrm{Na}_{2} \mathrm{SO}_{4}\left(0.9\right.$ mole $\mathrm{Na}_{2} \mathrm{SO}_{4}$; 10 moles $\mathrm{H}_{2} \mathrm{O}$ ) and an excess of solid $\mathrm{Na}_{2} \mathrm{~B}_{4} \mathrm{O}_{7} 10 \mathrm{H}_{2} \mathrm{O}$, dispersed within an emulsifying medium has been studied by differential scanning calorimetry. Two maxima of crystallization are observed at $+7{ }^{\circ} \mathrm{C}$ and $-2.5^{\circ} \mathrm{C}$ (without borax, the crystallization occurs around $-41^{\circ} \mathrm{C}$ ). Between the first and thirtieth cooling and heating cycles, an increase of about $15 \%$ of the calorific energy released during the crystallization has been found. This type of nucleation process could be of interest in the field of heat storage.
\end{abstract}

1. Introduction. - Par fusion d'une mole de $\mathrm{Na}_{2} \mathrm{SO}_{4}, 10 \mathrm{H}_{2} \mathrm{O}$ qui s'effectue à $32,4^{\circ} \mathrm{C}$ sous la pression atmosphérique, on obtient une solution contenant $33,3 \mathrm{~g}$ de sel anhydre pour $100 \mathrm{~g}$ de solution et du sel anhydre en excès. Cette transition s'accompagne d'une variation d'enthalpie positive de 58 calories par gramme de sel hydraté.

Lors du refroidissement du mélange obtenu si aucun germe de sel hydraté ne subsiste le système présente une sursaturation et de plus quand un germe est apparu il se développe lentement en l'absence d'agitation. L'action nucléante du borax : $\mathrm{Na}_{2} \mathrm{~B}_{4} \mathrm{O}_{7}$, $10 \mathrm{H}_{2} \mathrm{O}$ a fait l'objet de nombreux travaux. Ainsi pour des solutions saturées en sulfate de sodium et tétraborate de sodium à la température de transition et contenues dans des tubes de verre, le degré de sursaturation maximum observé est de l'ordre de $2{ }^{\circ} \mathrm{C}$. Pour des tubes ne contenant pas de borax le degré de sursaturation est de $15^{\circ} \mathrm{C}$ [1].

Du fait de l'importante énergie calorifique mise en jeu au cours de la transition, le mélange solide : $\mathrm{Na}_{2} \mathrm{SO}_{4}, 10 \mathrm{H}_{2} \mathrm{O}+$ borax a été proposé comme matériau de stockage thermique. Mais sous forme macroscopique le rendement du stockage baisse à chaque cycle du fait de l'agglomération à chaque fusion du sel anhydre. La réalisation de gels visant à augmenter l'interface $\mathrm{Na}_{2} \mathrm{SO}_{4}$-solution n'a pas apporté une amélioration sensible du rendement $[2,3,4]$.

A la lumière des études effectuées sur des gouttes de solution de quelques microns de diamètre dispersées au sein d'une émulsion et qui ont montré que les équilibres sel-solution s'établissent rapidement $[5,6,7]$, on peut penser améliorer le rendement en utilisant des émulsions. Dans ce but nous nous sommes proposés d'étudier l'action nucléante du borax pour des gouttes de solution de $\mathrm{Na}_{2} \mathrm{SO}_{4}$ de concentration légèrement inférieure à celle représentant une mole $\mathrm{Na}_{2} \mathrm{SO}_{4}$ pour 10 moles d'eau.

2. Expérimentation. - Les émulsions contenant des gouttes dont la concentration est de l'ordre de 1 mole de $\mathrm{Na}_{2} \mathrm{SO}_{4}$ pour 10 moles d'eau (44 g de $\mathrm{Na}_{2} \mathrm{SO}_{4}$ pour $100 \mathrm{~g}$ de solution) ne peuvent être préparées directement. On réalise tout d'abord une émulsion d'une solution sous-saturée contenant $30 \mathrm{~g}$ de $\mathrm{Na}_{2} \mathrm{SO}_{4}$ 
et $5 \mathrm{~g}$ de borax pour $100 \mathrm{~g}$ de solution au sein d'un support d'huile de paraffine associée à un agent émulsionnant favorisant les émulsions de type $\mathrm{E} / \mathrm{H}$. On obtient une émulsion de consistance pâteuse (viscosité de l'ordre de 14 poises à $20^{\circ} \mathrm{C}$ ) et dont le diamètre des gouttes est de l'ordre du micron. Il est ensuite possible d'évaporer à $30{ }^{\circ} \mathrm{C}$ par exemple la solution dispersée sans précipitation de sel au sein des gouttes jusqu'à une concentration voisine de celle du sel hydraté solide ( $41 \%$ par exemple).

L'émulsion ainsi obtenue placée dans un enthalpimètre (Perkin Elmer D.S.C. 2) est soumise à un refroidissement continu $\left(5^{\circ} \mathrm{C}\right.$. min. $\left.^{-1}\right)$ au cours duquel on observe des cristallisations prépondérantes autour de $-46^{\circ} \mathrm{C}$. $\mathrm{Au}$ cours du réchauffement suivant $\left(5^{\circ} \mathrm{C} . \mathrm{min}^{-1}\right)$ on observe une faible fusion eutectique et une transition à la température de $31^{\circ} \mathrm{C}$.

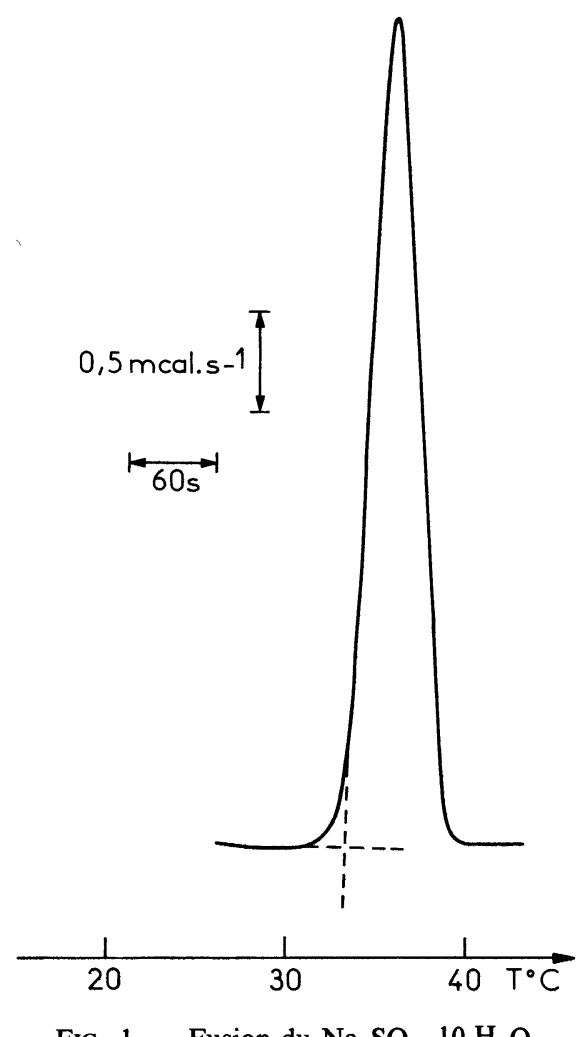

Fig. 1. - Fusion du $\mathrm{Na}_{2} \mathrm{SO}_{4}, 10 \mathrm{H}_{2} \mathrm{O}$.

[Melting of $\mathrm{Na}_{2} \mathrm{SO}_{4}, 10 \mathrm{H}_{2} \mathrm{O}$.]

$\mathrm{Au}$ cours du refroidissement ultérieur $\left(5^{\circ} \mathrm{C}\right.$ min..$\left.^{-1}\right)$ on observe la rupture de sursaturation entre $+12^{\circ} \mathrm{C}$ et $-7^{\circ} \mathrm{C}$ avec deux maxima de cristallisations à $+7{ }^{\circ} \mathrm{C}$ et $-2,5^{\circ} \mathrm{C}$.

L'ensemble de ces deux pics exothermiques représente une énergie calorifique qui est de l'ordre de $70 \%$ de celle du pic endothermique observé à $+31^{\circ} \mathrm{C}$ lors du réchauffement.

La première cristallisation à $-46^{\circ} \mathrm{C}$ représentant un taux élevé de sursaturation prouve que le borax lui-même est demeuré aussi en sursaturation. Il est à noter qu'en l'absence de borax la rupture de sur-
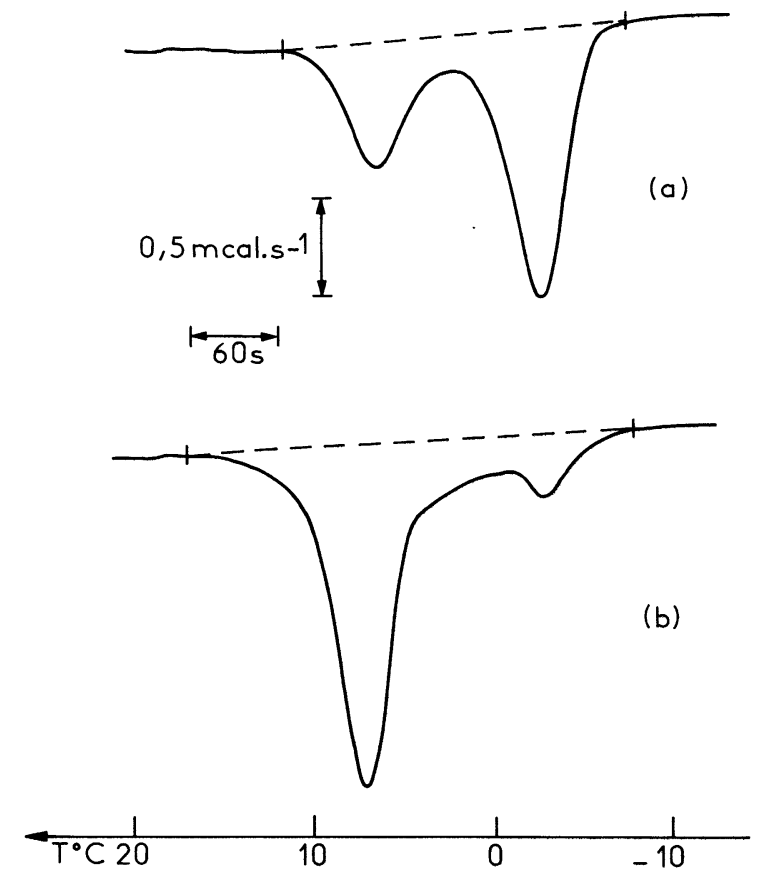

FIG. 2. - Cristallisation du $\mathrm{Na}_{2} \mathrm{SO}_{4}, 10 \mathrm{H}_{2} \mathrm{O}$ : a) $1^{\text {er }}$ cycle ; b) $30^{\mathrm{e}}$ cycle.

[Crystallization of $\mathrm{Na}_{2} \mathrm{SO}_{4}, 10 \mathrm{H}_{2} \mathrm{O}: a$ ) 1st cycle; $b$ ) 30th cycle.]

saturation s'effectue aux environs de $-41^{\circ} \mathrm{C}$ [7]. $\mathrm{La}$ fusion eutectique faiblement marquée indique que la totalité de l'eau, lors de la rupture de sursaturation n'a pas été incorporée au sel sous forme d'eau de cristallisation.

L'apparition, au deuxième refroidissement, de deux pics de cristallisation implique une action nucléante du borax qui a précipité au cours du premier refroidissement et a été dissous partiellement au cours du réchauffement. La quantité de borax introduite a été déterminée afin qu'il en soit ainsi. L'action nucléante du borax a été vérifiée par étude d'une émulsion ne contenant pas de borax et pour laquelle, au second refroidissement, on observe un degré de sursaturation très important, identique à celui observé au cours du premier refroidissement.

L'existence de deux pics de cristallisation au refroidissement manifeste un comportement différent pour deux populations de gouttes. Ce résultat pourrait être attribué à l'existence de diamètres de gouttes différents, mais alors ce phénomène devrait être également observé lors de la première cristallisation. Il semble plutôt que l'activité du borax ne soit pas identique dans toutes les gouttes. L'activité nucléante des microcristaux de borax serait alors répartie selon une loi de distribution en fonction de la température qui précisément se traduirait par la forme des pics. Il apparait par ailleurs que le borax soit moins actif dispersé dans des gouttes qu'en phase macroscopique. Cependant dans ce dernier cas la totalité du liquide cristallise dès que les premières nucléations dues à un nombre qui peut être relativement faible, 
de centres actifs du borax ont été déclenchées. En émulsion elles intéressent trop peu de gouttes pour être détectées. Il est également possible que le borax obtenu après une forte sursaturation soit moins actif.

Trente cycles successifs de refroidissement-réchauffement ont été effectués entre $+40^{\circ} \mathrm{C}$ et $-15^{\circ} \mathrm{C}$. On constate que les phénomènes se reproduisent avec une augmentation non négligeable du stockage, le rapport des aires de cristallisations observées aux $30^{\mathrm{e}}$ et 1 er cycles étant de 1,16 .

On remarque également une augmentation des cristallisations se produisant vers $+7{ }^{\circ} \mathrm{C}$ ce qui pourrait signifier une amélioration du pouvoir nucléant du borax. On peut conclure par ailleurs à une bonne conservation de l'émulsion au cours des trente cycles car des coalescences ne pourraient que causer une diminution du rendement.

3. Conclusion. - Ces phénomènes sont intéressants du point de vue de l'étude fondamentale de la nucléation spontanée ou provoquée. Ils paraissent être dignes d'approfondissement sur ce plan fondamental mais également dans le domaine de leur application au stockage thermique. Les gouttes sont le siège d'une cinétique de cristallisation suffisamment rapide pour permettre dans de bonnes conditions la récupération de l'énergie thermique stockée. De plus on constate un rendement qui tend à s'améliorer au cours des 30 cycles effectués. Cependant un tel usage nécessite la réalisation dans les conditions d'utilisation du matériau d'un nombre de cycles très élevé (de l'ordre du millier) pour lesquels le rendement se conserve de façon satisfaisante. Il faut également s'assurer de la conservation du matériau sur plusieurs années. Mais dès lors le principe même du type de dispersion des systèmes proposés ci-dessus peut être étendu à beaucoup d'autres sels, voire de corps organiques.

\section{Bibliographie}

[1] TelKes, M., Ind. Eng. Chem. 44 (1952) 1308.

[2] Tel.Kes, M., Ashrae J. (1974) 38.

[3] Farge Coulombier, M., Mater. Sci. Eng. Workshop on Energy Storage (Spring Quarter) 1976, p. 204.
[4] LoRsch, H., Thermal Energy Storage. Summary of University of Pennsylvania Studies (1976)

[5] BABIN, L., Thèse Bordeaux (1966).

[6] Clausse, D., J. Chim. Phys. 73 (1976) 333.

[7] Xans, P., C.R. Hebd. Séan. Acad. Sci. 177B (1973) 533. 